\title{
Revisión
}

\section{Revisión bibliográfica sobre características sociodemográficas y repercusiones de la depresión en el trabajador}

\author{
Literature review: socio-demographic characteristics \\ and impact of depression in the worker
}

\author{
John Jairo Córdoba Coronel1, 4, Alejandro Fuentes Fuentes'2, 4, Carolina Ruiz Rincón 3, 4 \\ 1. Hospital de Fuenlabrada-Madrid. Unidad Docente de Salud laboral de Madrid. \\ 2. Instituto Navarro de Salud laboral. Unidad Docente de Salud laboral de Navarra. \\ 3. Mutua Universal-Vigo. Unidad Docente de Salud Laboral de Galicia. \\ 4. Escuela Nacional de Medicina del Trabajo. Instituto de Salud Carlos III Madrid. España.
}

Recibido: 19-05-11

Aceptado: 13-06-11

\section{Correspondencia}

Carolina Ruiz Rincón

c/Cristo, 26, 3-A

36205 Vigo (Pontevedra). España

Teléfono: 627059648

Correo electrónico: caro.2.r@hotmail.com

Resumen

Introducción: La depresión es una enfermedad con efectos desfavorables en el lugar de trabajo. Ha sido asociada a discapacidad en términos de disminución de la productividad, absentismo, rotación laboral, y jubilación anticipada, entre otras.

Objetivos: Identificar las principales características sociodemográficas en los trabajadores con depresión.

Identificar el efecto que produce la depresión en el entorno laboral.

Metodología: Revisión bibliográfica en diferentes bases de datos, en dos fases: búsqueda/selección de artículos y evaluación de los 18 estudios seleccionados.

Resultados: Las mujeres, el bajo apoyo social, el estrés laboral, la inseguridad laboral y la insatisfacción laboral, entre otros, se asocian con mayor riesgo de depresión.

Mujeres con bajo nivel de autoridad, empleadas del área industrial o trabajos de alta demanda emocional, y los hombres con alto estrés, alta inseguridad laboral, los trabajos pasivos, están asociados con el riesgo de depresión.

La depresión causa absentismo laboral. Se estima que el bajo rendimiento en el trabajo produce pérdida de 225 millones de días de trabajo y $\$ 36.6$ billones de dólares por pérdida de productividad al año asociado a depresión.

Conclusiones: Existen diferencias por género en la asociación de síntomas depresivos y características psicosociales; estas podrían ser explicadas por la estructura de las ocupaciones, las características del trabajo de hombres y mujeres o por causas emergentes aún no determinadas, las cuales merecen futuras investigaciones. 
La relación entre la depresión, factores asociados y las repercusiones socioeconómicas a nivel laboral, señalan la necesidad de políticas de prevención y control en el trabajo.

Med Segur Trab (Internet) 2011; 57 (223) 174-187

Palabras claves: Absentismo. Depresión. Trastorno depresivo. Epidemiología. Prevalencia. Trabajador. Puesto de trabajo.

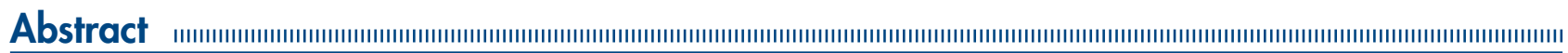

Introduction: Depression is an illness with undesirable effects in the work place. It has been associated with disability in terms of less productivity, absenteeism, labor rotation and anticipated retirement amongst others.

Objectives: Identify the main sociodemographic characteristics in workers with depression.

Identify the effects produced by depression in the workplace.

Methodology: Bibliographic review in different databases in two phases: search/selection of articles and review of the 18 selected studies.

Results: There is an increased risk of depression associated to women, low social support, workplace stress, job insecurity, job dissatisfaction, amongst others.

Business women with low level of authority, employees of the industrial area or emotional high demand jobs, as well as men with high stress, high job insecurity and passive jobs are associated with the risk of depression.

Depression causes absenteeism. It is estimated that poor performance at work causes the loss of 225 million workdays and $\$ 36.6$ billion dollars for productivity loss per year due to depression.

Conclusions:There are gender differences in the association of depressive symptoms and psychosocial characteristics; these could be explained by the structure of occupations, the characteristics of work carried out by men and women or emerging causes not yet determined which deserve further research.

The relationship between depression, associated factors and labor socioeconomic impacts, indicate the need for prevention and control policies at work.

Med Segur Trab (Internet) 2011; 57 (223) 174-187

Key words: Absenteeism, Depression, Depressive disorder, Epidemiology, Prevalence, Worker, Workplace. 


\section{INTRODUCCIÓN}

Actualmente los trastornos depresivos son la cuarta causa de discapacidad en el mundo ${ }^{1}$ y se espera que se conviertan en la segunda causa principal de morbilidad a nivel mundial ${ }^{2}$.

El estrés de origen laboral es el segundo problema de salud más frecuente relacionado con el trabajo en la unión europea, después de las enfermedades musculoesqueléticas ${ }^{1}$.

En la vida contemporánea, muchas ocupaciones se asocian con importantes exigencias mentales y emocionales que requieren habilidades interpersonales y personales para hacer frente a estas demandas ${ }^{3}$.

La depresión es un desorden común que parece tener un efecto muy desfavorable en el lugar de trabajo. En Estados Unidos, algunos estudios han reportado que el Trastorno Depresivo Mayor se asocia con 27 veces más probabilidad de pérdida de trabajo que entre los trabajadores sin un trastorno mental y que el $44 \%$ de los trabajadores deprimidos reportaron que ellos perdieron uno o más días de trabajo por problemas emocionales en los últimos 3 meses ${ }^{4}$.

Existen estudios en los que describen que los trabajadores con historia de depresión que no tuvieron un episodio reciente, no presentaron la misma disminución en la ejecución del trabajo como aquellos que presentaron episodios recientes, sugiriendo que la resolución de la depresión está asociada con la resolución de la incapacidad laboral. ${ }^{4}$

Algunos estudios de revisión han mostrado que las condiciones psicosociales del trabajo, especialmente la alta demanda y el bajo apoyo social son predictores de aparición de la depresión. Así mismo, diversos estudios han mostrado asociaciones entre factores psicosociales del entorno laboral, tales como el desequilibrio en la recompensa, la injusticia organizacional, conflictos interpersonales y los síntomas depresivos. ${ }^{5}$. Otros estudios han encontrado que el lugar de trabajo, el estrés laboral, la inseguridad laboral y el poco apoyo social en el lugar de trabajo, pueden precipitar el desarrollo de la depresión ${ }^{6,7}$.

La depresión se ha asociado con discapacidad en términos de disminución de la productividad ("presentismo"), el absentismo, la rotación laboral y el desempleo, así como la jubilación anticipada, baja calidad de vida y mayor riesgo de infarto de miocardio ${ }^{5}$.

Investigaciones relacionan el trabajo con las características psicosociales basadas en el modelo control-demandas-soporte e indican que la falta de control en el trabajo, la poca autoridad de decisión, baja cualificación y la tensión laboral (una combinación de alta demanda y bajo control) se asocian con el riesgo de depresión, ansiedad, angustia, fatiga, insatisfacción laboral y agotamiento ${ }^{8}$.

Los conflictos familiares que afectan el trabajo, pueden ser un obstáculo para el éxito y para satisfacer las demandas en el trabajo y las responsabilidades, lo que disminuye la capacidad de una persona para construir y mantener un ambiente positivo relacionado con el trabajo?.

La depresión mayor tiene un efecto significativo en la función laboral. En el 2010, se publicó que en Estados Unidos los trabajadores con depresión estiman un costo de 44.01 billones de dólares por año en pérdidas de productividad, un exceso de 30.94 billones de dólares por año en comparación con un costo previsto de los trabajadores sin depresión.

Los costes de la depresión no solo incluyen la discapacidad, el tratamiento, el absentismo y los costos administrativos, sino también el desempeño en el trabajo o "presentismo"10,11. 


\section{OBJETIVOS}

- Identificar las principales características sociodemográficas de los trabajadores con depresión.

- Identificar el efecto que produce la depresión en el entorno laboral.

\section{METODOLOGÍA}

Se procedió a la búsqueda de los artículos en las principales bases de datos del ámbito sanitario y a su posterior selección, según una serie de criterios de inclusión previamente establecidos.

Las bases de datos utilizadas fueron: PUBMED, CISDOC, OSH-UPDATE, WOK, IBECS, LILACS. En la estrategia de búsqueda se utilizaron combinaciones booleanas (and, or) de los siguientes términos:

Workplace, depressive disorder, management, occupational health, depression/ prevention and control, depressive disorder/drug therapy, depressive/epidemiology, absenteeism, prevalence, worker, stress, psychological.

Los criterios de inclusión establecidos fueron los siguientes:

- Artículos que tratan variables sociodemográficas relacionadas con la depresión en trabajadores.

- Artículos que proporcionan datos del impacto socioeconómico de la depresión en el trabajo.

Los criterios de exclusión fueron:

- Artículos de depresión en la embarazada, depresión posparto o en la población infantil.

- Artículos enfocados en el manejo farmacológico de la depresión.

- Artículos que no cumplen los criterios de inclusión.

Los límites de búsqueda empleados fueron:

- Periodo de publicación de los artículos: año 2005 a 2010.

- Población adulta mayor de 19 años.

- Idioma: sin restricciones.

La última fecha de búsqueda en las bases de datos fue el 14 de enero de 2011.

La búsqueda en las bases de datos electrónicas se completó con una búsqueda manual a partir de las listas de referencias de los artículos seleccionados.

La selección de los artículos se realizó en un primer tiempo a través de la lectura de los resúmenes de éstos, luego se descartaron los redundantes. Posteriormente se procedió a la lectura del texto íntegro de los artículos y basados en criterios de pertinencia (inclusión) se seleccionaron los artículos definitivos.

Los artículos elegidos inicialmente para el estudio fueron 394; de ellos se eliminaron posteriormente 376 porque no cumplían los requisitos establecidos, quedando por tanto seleccionados definitivamente para el estudio un total de 18 artículos (tabla 1). 
Tabla 1. Resultados de selección de artículos obtenidos en las diferentes bases de datos

\begin{tabular}{|c|c|c|c|c|c|c|}
\hline $\begin{array}{c}\text { Base } \\
\text { de } \\
\text { datos }\end{array}$ & $\begin{array}{l}\text { Términos incluidos en la } \\
\text { estrategia de búsqueda }\end{array}$ & $\begin{array}{c}\text { Número } \\
\text { de artículos } \\
\text { obtenidos } \\
\text { con los términos } \\
\text { de búsqueda }\end{array}$ & $\begin{array}{c}\text { Número } \\
\text { de artículos } \\
\text { redundantes }\end{array}$ & $\begin{array}{c}\text { Número } \\
\text { de artículos } \\
\text { seleccionados } \\
\text { por resúmen } \\
\text { (abstract) }\end{array}$ & $\begin{array}{c}\text { Número } \\
\text { de artículos } \\
\text { que no cumplen } \\
\text { con los criterios } \\
\text { de inclusión }\end{array}$ & 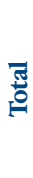 \\
\hline \multirow{5}{*}{ 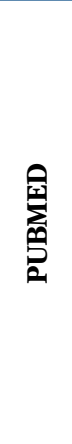 } & $\begin{array}{l}\text { WORKPLACE, DEPRESSIVE } \\
\text { DISORDER, MANAGEMENT }\end{array}$ & 35 & 0 & 7 & 3 & 4 \\
\hline & $\begin{array}{l}\text { Occupational, depression/ } \\
\text { prevention, control }\end{array}$ & 5 & 0 & 1 & 0 & 1 \\
\hline & $\begin{array}{l}\text { Depressive disorder/drug } \\
\text { therapy, depressive disorder/ } \\
\text { epidemiology, WORKPLACE }\end{array}$ & 24 & 5 & 11 & 4 & 7 \\
\hline & $\begin{array}{l}\text { ABSENTEEISM, } \\
\text { DEPRESSION, WORKPLACE }\end{array}$ & 6 & 1 & 1 & 0 & 1 \\
\hline & $\begin{array}{l}\text { Prevalence, depression, } \\
\text { WORKPLACE }\end{array}$ & 13 & 2 & 5 & 3 & 2 \\
\hline \multirow{3}{*}{ ర్ } & $\begin{array}{l}\text { Workplace, depressive } \\
\text { disorder, management }\end{array}$ & 2 & 0 & 1 & 0 & 1 \\
\hline & $\begin{array}{l}\text { Occupational health, } \\
\text { depression, management }\end{array}$ & 3 & 0 & 0 & 0 & 0 \\
\hline & $\begin{array}{l}\text { Depression, prevalence, } \\
\text { workplace }\end{array}$ & 4 & 0 & 0 & 0 & 0 \\
\hline \multirow{2}{*}{ 䆦 } & $\begin{array}{l}\text { Depressive disorder, } \\
\text { workplace, management }\end{array}$ & 1 & 1 & 0 & 0 & 0 \\
\hline & $\begin{array}{l}\text { Depression, management, } \\
\text { worker }\end{array}$ & 215 & 0 & 0 & 0 & 0 \\
\hline \multirow{2}{*}{ ○ै } & $\begin{array}{l}\text { Depression, management, } \\
\text { workplace }\end{array}$ & 57 & 1 & 3 & 2 & 1 \\
\hline & $\begin{array}{l}\text { Depressive disorder, } \\
\text { treatment, workplace }\end{array}$ & 8 & 2 & 1 & 1 & 0 \\
\hline \multirow{2}{*}{ 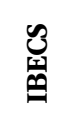 } & Absenteeism, depression & 5 & 0 & 3 & 3 & 0 \\
\hline & $\begin{array}{l}\text { Prevalence, depression, } \\
\text { stress, psychological }\end{array}$ & 3 & 0 & 1 & 0 & 1 \\
\hline \multirow{2}{*}{ 岁 } & WORKPLACEDEPRESSIVE & 6 & 0 & 1 & 1 & 0 \\
\hline & Absenteeism, Depression & 7 & 0 & 3 & 3 & 0 \\
\hline
\end{tabular}




\section{RESULTADOS}

La tabla 2 resume los dieciocho artículos analizados (doce de diseño tipo cohortes, tres transversales, uno observacional descriptivo, un ensayo controlado aleatorio y uno de casos-controles) relativos a características sociodemográficas del trabajadores con depresión y sus repercusiones en el entorno laboral.

Tabla 2a. Características sociodemográficas

\begin{tabular}{ccc}
\hline $\begin{array}{c}\text { Autor } \\
\text { Año }\end{array}$ & Tipo & Tamaño de muestra \\
de estudio & y contexto & Resultados \\
\hline
\end{tabular}

Rodríguez B, et Observacional $\mathrm{n}=1628$ trabajadores en - Un total de 1.628 trabajadores en IT (11,2\%)

al. 2005(1) descriptivo Incapacidad Temporal presentaban como diagnóstico principal algún (IT) de laComunidad trastorno mental, de los cuales el 17,8\% (289) Autónoma de Madrid. relacionan su patología con el trabajo. La edad media fue de 43,5 años. Las mujeres presentan con mayor frecuencia T. mental como causa de IT. - Los trabajadores cualificados: directivos (41\%), técnicos científico-intelectuales (30\%) y empleados administrativos (27\%) son los que presentan mayor prevalencia de sintomatología depresiva asociada al trabajo.

Nieuwenhuijsen Cohorte K, et al. 2006

(12)

Rugulies R, et al. Cohorte 2006(13)

Blackmore ER, Cohorte et al. 2007 (2) $\mathrm{n}=188$ trabajadores Países Bajos

Dinamarca, entrevista telefónica. $n=4133$ trabajadores ( $48,5 \%$ Mujeres).

Canadá, n= 24324 trabajadores, encuesta, ponderado a $\mathrm{n}=745948$.
- Predictores negativos para la depresión: la edad mayor de 50 años [HR 0,5, 95\% intervalo de confianza (IC del 95\%) 0.3-0,8], sexo masculino, las expectativas negativas de recuperación (HR 0.5, IC 95\%: 0.3-0.8), un medio o alto nivel de la educación (HR 0.5, IC 95\%: 0,3 a 0.8).

- Depresión, correlación ( + ) coeficiente correlación $0.31(\mathrm{p}<0.001)$.

- Síntomas de depresión severa en 68 mujeres, 37 hombres. (105).

- Incidencia 2.5 (3.4 mujeres y 1,7 hombres).

- En Mujeres: los factores fuertemente asociados con los síntomas de depresión severa son:

- Influencia del trabajo $(\mathrm{RR})=2.17,95 \%$

$(\mathrm{IC})=1.23,3.82$.

- Escaso apoyo del supervisor: $(\mathrm{RR})=2.03$, $95 \%$ (IC) $=1.2,3.43$ ).

- En hombres: La inseguridad laboral (RR)= $2.04,95 \%$ (IC) $=1.02-4.06$

- Depresión en el 4.6\% (745948) general. Mayor en mujeres $6 \%(\mathrm{OR})=1.83(1.53,2.2)$, comparado con el de hombres $3.4 \%$.

- Mujeres: con bajo nivel de autoridad tienen una mayor probabilidad de depresión ([OR]= $1.59 ; 95 \%$ (IC) $=1.06,2.39$ ), que los hombres en condiciones similares.

- Hombres: con alto estrés laboral y/o inseguridad laboral tienen mayor probabilidad de depresión que las mujeres.

- Estrés laboral $[\mathrm{OR}]=2.38 ; 95 \%$ (IC) $=1.29,4.37$.

- Inseguridad laboral $(\mathrm{OR})=2.66 ; 95 \%$ (IC) $=$ 1.56, 2.66 .

- En hombres y mujeres: el poco apoyo social se asocia a mayor probabilidad de depresión. Hombres: $[\mathrm{OR}]=2.7 ; 95 \%$ (IC) $=1.55,4.71$; Mujeres: $[\mathrm{OR}]=2.37 ; 95 \%(\mathrm{IC})=1.71,3.29$. 


\begin{tabular}{|c|c|c|c|}
\hline $\begin{array}{l}\text { Autor } \\
\text { Año }\end{array}$ & $\begin{array}{c}\text { Tipo } \\
\text { de estudio }\end{array}$ & $\begin{array}{l}\text { Tamaño de muestra } \\
\text { y contexto }\end{array}$ & Resultados \\
\hline $\begin{array}{l}\text { Demir F, et al. } \\
2007(14)\end{array}$ & Transversal & $\begin{array}{l}\mathrm{n}=156 \text { residentes de } \\
\text { medicina en Estambul, } \\
\text { con una edad promedio } \\
\text { de } 28.2 \text { años. }\end{array}$ & $\begin{array}{l}\text { - La prevalencia de probable depresión entre } \\
\text { los residentes fue de } 16 \% \text { (IC:95\%: } 10.7-22.7 \text { ). } \\
\text { - La prevalencia de depresión entre los } \\
\text { primeros años de residencia fue más alta } \\
(22.2 \%) \text { comparada con los residentes } \\
\text { mayores }(12.7 \%) \text {, pero la diferencia no fue } \\
\text { estadísticamente significativa ( }>0.05 \text { ). } \\
\text { - Tomando el género masculino como } \\
\text { referencia, la OR para la mujer fue } 5.16 \text { (95\% IC: } \\
1.51-17.68, p<0.01) \text {. }\end{array}$ \\
\hline $\begin{array}{l}\text { Kessler RC, et } \\
\text { al. } 2008 \text { (15) }\end{array}$ & Cohorte & $\begin{array}{l}\mathrm{n}=3378 \text { personas } \\
\text { mayores de } 18 \text { años, } \\
\text { de los Estados Unidos. }\end{array}$ & $\begin{array}{l}\text { - El } 6.4 \% \text { de los empleados, presentaron } \\
\text { trastorno de depresión mayor. } \\
\text { - La prevalencia del trastorno depresivo } \\
\text { mayor en los últimos } 12 \text { meses no difiere } \\
\text { significativamente en la educación, ocupación o } \\
\text { las horas de trabajo, pero es significativamente } \\
\text { más alta en las mujeres que en hombres y está } \\
\text { inversamente relacionada con la edad. } \\
\text { - Se reportó una mayor prevalencia de } \\
\text { trastorno depresivo mayor en los jóvenes } \\
\text { trabajadores en comparación con los mayores. }\end{array}$ \\
\hline $\begin{array}{l}\text { Wege N, et al. } \\
2008 \text { (16) }\end{array}$ & Cohorte & $\begin{array}{l}\text { Alemania, } \mathrm{n}=1749 \\
\text { empleados y/o } \\
\text { autónomos. Entrevistas } \\
\text { y cuestionarios. } \\
\text { Trabajadores }>15 \mathrm{~h} / \\
\text { Semana. }\end{array}$ & $\begin{array}{l}\text { - La clase social baja se relaciona en } \\
\text { hombres y en mujeres con incidencia de } \\
\text { depresión; (OR)= } 2.61-8.2 ; 95 \% \text { (IC) }= \\
1.53,14.15 \text {. }\end{array}$ \\
\hline $\begin{array}{l}\text { Kouvonen A, et } \\
\text { al. } 2008 \text { (17) }\end{array}$ & Cohorte & $\begin{array}{l}\text { Finlandia, } \mathrm{n}=33.577 \\
\text { trabajadores en el } \\
\text { sector público. }\end{array}$ & $\begin{array}{l}\text { - El bajo capital social está relacionado con } \\
\text { mayor riesgo de depresión auto percibida. }\end{array}$ \\
\hline $\begin{array}{l}\text { Wieclaw J, et al. } \\
2008 \text { (8) }\end{array}$ & $\begin{array}{l}\text { Casos y } \\
\text { controles }\end{array}$ & $\begin{array}{l}\text { Dinamarca, } \\
\text { Casos (depresión } \\
\text { y/o desordenes } \\
\text { de ansiedad, } \\
\text { diagnosticados por } \\
\text { psiquiatra): } 14166, \text { y } \\
\text { Controles: } 58060 .\end{array}$ & $\begin{array}{l}\text { - } 33 \% \text { de los casos con diagnostico de } \\
\text { desorden depresivo. } \\
-53 \% \text { de depresión en }>\text { de } 40 \text { años. } \\
- \text { Tener bajos ingresos y vivir a las afueras de } \\
\text { una ciudad grande están asociados a riesgo de } \\
\text { depresión. } \\
\text { - El alto riesgo de depresión en mujeres } \\
\text { se relaciona con trabajos que tienen altas } \\
\text { demandas emocionales IRR }=1.39 ; 95 \% \text { (IC): } \\
1.22,1.58 \text {, atención al cliente: IRR }=1.15 ; 95 \% \\
\text { (IC): } 1.01-1.3 \text {. } \\
\text { - Los niveles medio y medio-altos de trabajo } \\
\text { con la gente se relacionan con la disminución } \\
\text { significativa del riesgo de depresión IRR: } 0.85 ; \\
95 \% \text {, (IC) }=0.73 \text { a } 0.98 \text {. }\end{array}$ \\
\hline $\begin{array}{l}\text { Magnusson LL, } \\
\text { et al. } 2009 \text { (5) }\end{array}$ & Cohorte & $\begin{array}{l}\mathrm{n}=5985 \text { trabajadores } \\
\text { de Suecia, con edades } \\
\text { entre } 16 \text { y } 64 \text { años. }\end{array}$ & $\begin{array}{l}\text { - Un menor grado de decisión de la autoridad } \\
\text { fue asociado con los síntomas depresivos entre } \\
\text { hombres y mujeres. } \\
\text { - El más alto grado de apoyo en las mujeres } \\
\text { por parte de los superiores, estuvo asociado con } \\
\text { mayor puntaje en la escala de depresión. } \\
\text { - Los conflictos con compañeros de trabajo en } \\
\text { los hombres estuvieron asociados con aumento } \\
\text { de los síntomas depresivos. }\end{array}$ \\
\hline
\end{tabular}




\begin{tabular}{cccc}
\hline $\begin{array}{c}\text { Autor } \\
\text { Año }\end{array}$ & Tipo & Tamaño de muestra & Resultados \\
\hline
\end{tabular}

Sinokki M, et al. Cohorte 2009 (18)

Wang J, et al. Cohorte 2009 (19)

Clumeck N, et Cohorte al. $2009(20)$

Hämmig $\mathrm{O}$, et Transversal al. $2009(21)$ $\mathrm{n}=3347$ trabajadores - El pobre clima en el equipo de trabajo estuvo de Finlandia entre 30 y significativamente asociado con trastornos 64 años. depresivos (OR 1.61, IC 95\%: 1,10 a 2,36). Una mayor proporción de mujeres que de hombres tenían trastornos de depresión.

Canadá, n= 4866 trabajadores. Entrevistas. - Los trabajadores que presentaron alto estrés laboral persistente presentaron el doble de incidencia de depresión que el grupo de bajo estrés.

— Incidencia 8\%; OR: 1.52; 95\% (IC): 5.3 - 10.6

vs. $4 \%$; OR 1; $95 \%$ (IC): $2.8-5.2$.

- El incremento del estrés laboral presentó 1.6 veces más el desarrollo de depresión mayor que el grupo de bajo estrés.

- Trabajadores que disminuyeron el nivel de estrés laboral, tuvieron un riesgo de depresión mayor similar al grupo de bajo estrés. 4.4\%; OR 0.97; $95 \%$ (IC): 2.7 - 6.2 vs. $4 \%$; OR 1, 95\% (IC): $2.8-5.2$.

Bélgica, $\mathrm{n}=9396 \quad$ - La incidencia de depresión a largo plazo fue trabajadores. menor en hombres (1.4\%) comparado con el de las mujeres (3.4).

- La depresión en hombres fue más frecuente para los trabajadores de cuello blanco (1.6\%) y cuello azul (1.7\%) comparada con los ejecutivos $(0.6 \% ; \mathrm{p}=0.02)$.

- La depresión en mujeres fue notoriamente mayor en trabajadores de cuello azul (6.1\%) comparado con los de cuello blanco (3\%), y ejecutivos (3.9\%; $\mathrm{p}=0.04)$.

- En hombres se asoció positivamente el alto estrés laboral (OR= 4.8; 95\% IC: $2.1,11)$ y la pasividad (OR= 3.6; 95\% IC: $1.6,8.3)$, y el alto estrés/bajo apoyo social (OR=3.06; $95 \%$ IC: 1.71-5.5) con la incidencia de depresión.

- En mujeres el efecto del estrés no fue significativo ( $\mathrm{p}=0.13$ y 0.22 ).

Suiza, $n=4371$ trabajadores.
- Las tasas de prevalencia del conflicto laboral con la vida privada, son claramente superiores en hombres y mujeres con alta educación en ejecutivos o funcionarios de manejo, en trabajos de tiempo completo o con variación de horarios, contratos irregulares, largo tiempo de trayecto al trabajo e inseguridad laboral.

- Las horas extraordinarias (B 0.14; $\mathrm{p}<0.001)$ y los horarios de trabajo (B $0.12 ; \mathrm{p}<0.001)$ son los más fuertemente asociada con el conflicto del trabajo y la vida privada en hombres, asociados con la depresión.

- Las horas de trabajo (B: 0.28; $\mathrm{p}<0.001$ ) es el más fuertemente asociado con el conflicto del trabajo y la vida en las mujeres seguido de la posición de manejo (B: $0.09 ; \mathrm{p}<0.001$ ) y los horarios (B: 0.09; p<0.001). 
Tabla 2b. Repercusiones

\begin{tabular}{|c|c|c|c|}
\hline $\begin{array}{l}\text { Autor } \\
\text { Año }\end{array}$ & $\begin{array}{c}\text { Tipo } \\
\text { de diseño }\end{array}$ & $\begin{array}{l}\text { Tamaño de muestra } \\
\text { y contexto }\end{array}$ & Resultados \\
\hline $\begin{array}{l}\text { Lo Sasso AT, et } \\
\text { al. } 2006(22)\end{array}$ & $\begin{array}{l}\text { Ensayo } \\
\text { controlado } \\
\text { aleatorio }\end{array}$ & $\begin{array}{l}\text { Estados Unidos, } \\
\mathrm{n}=198 \text { trabajadores. }\end{array}$ & $\begin{array}{l}\text { - Demuestra que muchos empleadores } \\
\text { pueden recibir un rendimiento potencial } \\
\text { significativo de la inversión en el } \\
\text { tratamiento de la depresión de } 30 \text { dólares } \\
\text { por cada trabajador que es tratado durante } \\
\text { el 1er año y } 257 \text { dólares en el } 2 \text { año, con } \\
\text { lo cual se estima un retorno de la inversión } \\
\text { durante el periodo a } 2 \text { años del } 302 \% \text {; con } \\
\text { mejora en el absentismo y la productividad } \\
\text { en el trabajo. }\end{array}$ \\
\hline $\begin{array}{l}\text { Demir F, et al. } \\
2007 \text { (14) }\end{array}$ & Transversal & $\begin{array}{l}156 \text { residentes de } \\
\text { medicina en Estambul, } \\
\text { con una edad } \\
\text { promedio de } 28.2 \\
\text { años. }\end{array}$ & $\begin{array}{l}\text { - Se determinó una correlación negativa entre } \\
\text { la depresión y la satisfacción laboral }(\mathrm{r}=-0.36 \text {, } \\
\mathrm{p}<0.001) \text {. La correlación entre la satisfacción } \\
\text { laboral y la depresión fue más fuerte entre los } \\
\text { residentes de primer año }(\mathrm{r}=-0.50, \mathrm{p}<0.001) \text {. }\end{array}$ \\
\hline $\begin{array}{l}\text { Kessler RC, et } \\
\text { al. } 2008 \text { (15) }\end{array}$ & Cohorte & $\begin{array}{l}3378 \text { personas mayores } \\
\text { de } 18 \text { años, de los } \\
\text { Estados Unidos. }\end{array}$ & $\begin{array}{l}\text { - El TDM está asociado con una significativa } \\
\text { pérdida de rendimiento en el trabajo, se estiman } \\
225 \text { millones de días de trabajo y } \$ 36.6 \text { billones } \\
\text { por pérdida de productividad al año asociado } \\
\text { con TDM. La tasa superior de tratamiento en } \\
\text { los trabajadores parece reflejar una mayor } \\
\text { tasa de seguro de salud entre los trabajadores } \\
\text { deprimidos que entre los deprimidos sin trabajo. }\end{array}$ \\
\hline $\begin{array}{l}\text { Schwenk TL, et } \\
\text { al. } 2008 \text { (23) }\end{array}$ & Transversal & $\begin{array}{l}1154 \text { médicos de } \\
\text { Michigan. }\end{array}$ & $\begin{array}{l}\text { - Se reportó depresión moderada a severa en } \\
130 \text { médicos }(11.3 \%) \text {. } \\
\text { - Los médicos que reportaron depresión } \\
\text { moderada a severa fueron } 2 \text { a } 3 \text { veces más } \\
\text { propensos a reportar un impacto sustancial en } \\
\text { sus roles de trabajo en comparación con los } \\
\text { médicos con puntuaciones de depresión leve, } \\
\text { incluyendo una disminución de la productividad } \\
\text { en el trabajo ( } 57.7 \% \text { vs } 18.5 \% \text {; p<0.01) y una } \\
\text { disminución en la satisfacción laboral }(90.8 \% \text { vs } \\
36.2 \% \text {, p }<0.01) \text {. }\end{array}$ \\
\hline $\begin{array}{l}\text { Kouvonen A, et } \\
\text { al. } 2008 \text { (17) }\end{array}$ & Cohorte & $\begin{array}{l}\text { Finlandia, } \mathrm{n}=33.577 \\
\text { trabajadores en el } \\
\text { sector público. }\end{array}$ & $\begin{array}{l}\text { - Tratamiento antidepresivo: } 20-50 \% \text { superior } \\
\text { para empleados con escaso apoyo social. }\end{array}$ \\
\hline $\begin{array}{l}\text { Sinokki M, et al. } \\
2009 \text { (18) }\end{array}$ & Cohorte & $\begin{array}{l}3347 \text { trabajadores de } \\
\text { Finlandia entre } 30 \text { y } 64 \\
\text { años. }\end{array}$ & $\begin{array}{l}\text { - El pobre clima en el equipo de trabajo } \\
\text { predijo el uso de medicación antidepresiva (OR } \\
1.53 \text {, IC } 95 \%: 1,02 \text { a } 2,30 \text { ). }\end{array}$ \\
\hline $\begin{array}{l}\text { Clumeck N, et } \\
\text { al. } 2009(20)\end{array}$ & Cohorte & $\begin{array}{l}\text { Bélgica, } n=9396 \\
\text { trabajadores. }\end{array}$ & $\begin{array}{l}\text { - El absentismo por depresión fue superior en } \\
\text { hombres que en mujeres. } \\
-100 \text { hombres y } 100 \text { mujeres se ausentaron } \\
\text { más de } 28 \text { días por episodio de depresión. } \\
\text { - El bajo control laboral se asocio directamente } \\
\text { con el absentismo causado por depresión en } \\
\text { hombres (OR= } 3.38 ; 95 \% \text { IC: } 1.79,6.37) \text { y en } \\
\text { mujeres (OR= } 2.05 ; 95 \% \text { IC: } 1.04,4.03) \text {. }\end{array}$ \\
\hline
\end{tabular}




\begin{tabular}{|c|c|c|c|}
\hline $\begin{array}{l}\text { Autor } \\
\text { Año }\end{array}$ & $\begin{array}{c}\text { Tïpo } \\
\text { de diseño }\end{array}$ & $\begin{array}{l}\text { Tamaño de muestra } \\
\text { y contexto }\end{array}$ & Resultados \\
\hline $\begin{array}{l}\text { Sicras A, et al. } \\
2010(24)\end{array}$ & Cohorte & $\begin{array}{l}4572 \text { personas de } \\
\text { con diagnóstico } \\
\text { de depresión en } \\
\text { España. }\end{array}$ & $\begin{array}{l}\text { - La incapacidad por enfermedad en } \\
\text { pacientes con depresión que no remitió, } \\
\text { tuvo una media de } 38.5 \text { días, comparado } \\
\text { con el grupo en el que los síntomas } \\
\text { remitieron, que fue de } 31 \text { días, p<0.001. } \\
\text { - El coste sanitario bruto anual de los } \\
\text { pacientes que demandaron asistencia } \\
\text { durante los } 12 \text { meses de seguimiento } \\
\text { ascendió a } 12,3 \text { millones de euros. Los } \\
\text { costes totales desagregados en sanitarios } \\
\text { (directos)y pérdidas de productividad } \\
\text { laboral (no sanitarios, indirectos) fueron } \\
\text { del } 32,9 \% \text { y el } 67,1 \%, \text { respectivamente } \\
\text { (coste promedio/ unitario: } 888,5 \text { y } 1809,6 \\
€ ; \text { p }<0,02 \text { ). El coste total de la atención } \\
\text { sanitaria ascendió a } 8,3 \text { millones de euros, } \\
\text { de los cuales el } 40,6 \% \text { correspondió a } \\
\text { costes en atención especializada y el } \\
\text { restante } 59,4 \% \text { a atención primaria. El coste } \\
\text { medio por paciente de los costes directos } \\
\text { brutos en presencia o ausencia de remisión } \\
\text { fue de } 706,0 € \text { frente a } 1.108,3 € ; \text { p }<0,001 \text {. } \\
\text { En el modelo corregido, los costes directos } \\
\text { fueron de } 793,0 € \text { (IC95\%: } 739,4-846,7 € \text { ) } \\
\text { frente a } 1080,8 € \text { (IC95\%: } 1.020,3-1.141,4 \mathrm{~h} \text { ), } \\
\text { respectivamente; }<<0,001 \text {. } \\
\text { - Los costes por pérdida de productividad } \\
\text { laboral fueron de } 1.609,2 € \text { y de } 2.282,1 €, \\
\text { respectivamente; }<0,001 \text {. La duración } \\
\text { media del tratamiento antidepresivo } \\
\text { prescrito en cada grupo (remisión/no } \\
\text { remisión a los } 18 \text { meses) fue de } 146,6 \\
\text { frente a } 307,7 \text { dias; p<0,001 (mediana: } 112 \\
\text { frente a } 291,2 \text { días; p }<0,001 \text { ). }\end{array}$ \\
\hline $\begin{array}{l}\text { Wang JL, et al. } \\
2010 \text { (9) }\end{array}$ & Cohorte & $\begin{array}{l}4032 \text { empleados, } \\
\text { entre } 25 \text { y } 65 \text { años } \\
\text { de edad Alberta, } \\
\text { Canadá. }\end{array}$ & $\begin{array}{l}\text { - El desequilibrio entre la tensión laboral y } \\
\text { la recompensa al esfuerzo al parecer afecta el } \\
\text { rendimiento laboral a través de la gravedad de } \\
\text { la depresión. } \\
\text { - } 47,3 \% \text { - } 42,9 \% \text { reportó algún grado } \\
\text { de deterioro en el desempeño laboral, } \\
\text { siendo la depresión el factor más fuertemente } \\
\text { asociado. }\end{array}$ \\
\hline
\end{tabular}

Cuatro artículos trataron el tema de la edad; en dos de ellos se reporta una edad media de 43.5 años y una edad superior a 40 años como factor asociado a la depresión, a diferencia de los otros artículos que señalan la edad mayor de 50 años como predictor negativo y mayor prevalencia de trastorno depresivo mayor en los trabajadores jóvenes.

La variable sexo fue mencionada en ocho artículos, coincidiendo todos con la mayor prevalencia en mujeres. 
Siete artículos hicieron referencia al nivel educativo y/o cargo en la empresa (nivel de autoridad), con diversas conclusiones: en un estudio, los trabajadores cualificados presentaron una mayor prevalencia de sintomatología depresiva; en otro, un medio o alto nivel de la educación se relacionó como predictor negativo frente a la depresión. Hubo un estudio donde no hubo diferencia significativa entre la prevalencia de trastorno depresivo mayor en los últimos 12 meses y la educación u ocupación. En el estudio de Clumeck, et $\mathrm{al}^{20}$, la depresión en hombres fue más frecuente para los trabajadores de "cuello blanco (1)" y "cuello azul(2)" comparado con los ejecutivos; y en las mujeres, fue notoriamente mayor en trabajadoras de "cuello azul" comparado con las de cuello blanco, y ejecutivos. Un estudio reportó una prevalencia superior en hombres o mujeres con alta educación en ejecutivos o funcionarios de manejo.

Finalmente, dos artículos concluyeron que trabajadores con un bajo nivel de autoridad tienen mayor probabilidad de desarrollar depresión.

Tres artículos describen que la clase socioeconómica baja se relaciona con depresión.

Cinco estudios trataron el tema del apoyo social, bien sea por parte de sus supervisores o de sus compañeros de trabajo. Llama la atención que dos estudios difieren en sus resultados, ya que uno asegura que el escaso apoyo del supervisor está fuertemente asociado con los síntomas de depresión severa en mujeres, mientras que otro estudio señala que el más alto grado de apoyo en las mujeres por parte de los superiores, estuvo asociado con mayor puntaje en la escala de depresión. Los otros tres estudios coinciden en que el poco apoyo social o pobre clima laboral, estuvo asociado con trastornos de depresión.

Con respecto a la inseguridad laboral, tres artículos documentaron que ésta se asocia con la depresión; y en dos de estos estudios mencionan que esta variable afecta con mayor frecuencia a los hombres.

En lo relacionado al estrés laboral, cinco artículos reportan datos que concuerdan con que esta característica se asocia con la depresión, y en dos de ellos informan que hubo más prevalencia en hombres y en mujeres, respectivamente.

Sólo dos estudios hicieron alusión al horario de trabajo. En el primero no hubo diferencia significativa ente la depresión y el horario, pero en el segundo estudio se determinó que las horas extraordinarias y los horarios de trabajo están fuertemente asociados con el conflicto del trabajo y la vida privada en hombres con depresión.

Tres artículos mencionan el tratamiento de la depresión relacionado con otras variables: Un ensayo controlado aleatorizado demuestra que muchos empleadores pueden recibir un rendimiento potencial significativo de la inversión en el tratamiento de la depresión de 30 dólares por cada trabajador que es tratado durante el 1 . $^{\text {er }}$ año y 257 dólares en el $2 .^{\circ}$ año, con lo cual se estima un retorno de la inversión durante el periodo a 2 años del $302 \%$, con mejora en el absentismo y la productividad en el trabajo.

Los restantes dos estudios coinciden en que el pobre apoyo social predijo el mayor uso de antidepresivos.

En lo relacionado a las repercusiones de la depresión en cuanto a insatisfacción laboral, desempeño laboral, absentismo y coste, se contó con los resultados de seis de los dieciocho artículos y se obtuvo:

En dos estudios que contaron con población de médicos se encontró una correlación negativa entre la depresión y la satisfacción laboral, siendo más fuerte entre los residentes de primer año y en los médicos que reportaron depresión moderada a severa.

(1) Trabajadores de cuello blanco (white collar workers): Aquellos trabajadores que se encargan del área de "oficina", oficinistas, secretarias ${ }^{20}$.

(2) Trabajadores de cuello azul: Equivalente al término inglés "blue collar workers", corresponde a los trabajadores del área industrial, fábricas o de talleres (obreros, operarios, oficiales, mecánicos) ${ }^{20}$. 
En otro estudio informaron que el desequilibrio entre la tensión laboral y la recompensa al esfuerzo parece afectar el rendimiento laboral.

Un estudio realizado en Estados Unidos señala que el trastorno depresivo mayor está asociado con una significativa pérdida de rendimiento en el trabajo, con estimaciones de 225 millones de días de trabajo y $\$ 36.6$ billones por pérdida de productividad al año asociado con TDM. Mientras que otro estudio realizado en Bélgica afirma que el absentismo por depresión fue superior en hombres que en mujeres, entre los cuales 100 hombres y 100 mujeres se ausentaron más de 28 días por episodio de depresión; además, el bajo control laboral se asoció directamente con el absentismo causado por depresión en hombres y en mujeres.

Para terminar, cabe destacar un estudio realizado en España y publicado en el 2010 en el que informan que la incapacidad por enfermedad en pacientes con depresión que no remitió, tuvo una media de 38.5 días, comparado con el grupo en el que los síntomas remitieron, que fue de 31 días. El coste sanitario bruto anual de los pacientes que demandaron asistencia durante los 12 meses de seguimiento ascendió a 12,3 millones de euros. Los costes totales desagregados en sanitarios (directos) y pérdidas de productividad laboral (no sanitarios, indirectos) fueron del 32,9 \% y el 67,1 \%, respectivamente (coste promedio/unitario: 888,5 y $1809,6 €$ ). El coste total de la atención sanitaria ascendió a 8,3 millones de euros, de los cuales el 40,6 \% correspondió a costes en atención especializada y el restante $59,4 \%$ a atención primaria. El coste medio por paciente de los costes directos brutos en presencia o ausencia de remisión fue de 706,0€ frente a 1.108,3€. Los costes por pérdida de productividad laboral fueron de $1.609,2 €$ y de $2.282,1 €$, respectivamente. La duración media del tratamiento antidepresivo prescrito en cada grupo (remisión/no remisión a los 18 meses) fue de 146,6 frente a 307,7 días.

\section{CONCLUSIONES}

Basándonos en los artículos de cohorte y de casos y controles, los cuales destacan por ser de los mejores para el estudio de la multiefectividad del riesgo y de la multicausalidad, respectivamente. Podemos resaltar los predictores positivos para la depresión, como son: el sexo femenino, la inseguridad laboral, el bajo apoyo social, la baja remuneración económica, el pobre clima laboral, el desequilibrio entre la tensión laboral y la recompensa al esfuerzo, el bajo control laboral y vivir en las afueras de una ciudad grande.

Se evidencian diferencias por género importantes, así en mujeres el bajo nivel de autoridad, los trabajos de cuello azul del área industrial, fábricas o talleres, o los trabajos que tienen alta demanda emocional, están asociados con el riesgo de depresión. Mientras que en hombres son; el estrés, la inseguridad laboral, los trabajos pasivos, los trabajos de cuello blanco y azul, los más frecuentemente asociados con depresión. Estas diferencias podrían ser explicadas por la estructura de las ocupaciones, las características del trabajo, o por causas emergentes en el inicio de los síntomas depresivos, los cuales merecen futuras investigaciones.

El pobre clima laboral y el escaso apoyo social en trabajadores con depresión están asociados con la mayor necesidad de tratamiento farmacológico.

Resultados contradictorios los vistos en cuanto a la edad, el nivel de educación, la ocupación, el apoyo del supervisor, el estrés laboral y las horas de trabajo.

Dentro de los efectos de la depresión en el área laboral, se evidencia que produce el $50 \%$ de todo el deterioro del desempeño laboral, y con ello el alto coste. Se estima que del $100 \%$ de los costes totales (sanitarios y no sanitarios - por pérdida de productividad laboral-) que generan los pacientes con depresión, el $67 \%$ corresponde a las pérdidas de productividad laboral. 
Dentro de los artículos que mejor valoran la utilidad de una intervención y aportan mayor evidencia causal, se encuentran los ensayos clínicos. Lamentablemente se disponen de escasos ensayos clínicos, en este campo. El ensayo controlado aleatorizado de Lo Sasso, et al $^{22}$, evidencia el beneficio que los empleadores pueden recibir. Un rendimiento significativo invirtiendo en el tratamiento de la depresión de sus trabajadores. Un retorno de la inversión durante el periodo a 2 años del $302 \%$; con mejora del absentismo y de la productividad en el trabajo.

La relación entre la depresión, factores asociados y las repercusiones socioeconómicas a nivel laboral, señala la necesidad de políticas de prevención y control en el trabajo.

\section{REFERENCIAS BIBLIOGRÁFICAS}

1. Rodríguez B, Gómez A, García A, López I. Sintomatología depresiva asociada al trabajo como causa de incapacidad temporal en la Comunidad de Madrid. Mapfre Medicina 2005; 16(3):184-194.

2. Blackmore ER, Stansfeld SA, Weller I, Munce S, Zagorski BM, Stewart DE. Major depressive episodes and work stress: results from a national population survey. Am J Public Health 2007 Nov; 97(11):2088-93.

3. Lerner D, Adler DA, Chang H. Unemplyment, job retention, and productivity loss among employees with depression. Psychiatr Serv 2004; 55(12):1371-1378.

4. Kessler RC, Akiskal HS, Ames M, Birnbaum H, Greenberg P, Hirschfeld RM, et al. Prevalence and effects of mood disorders on work performance in a nationally representative sample of U.S. workers. Am J Psychiatry 2006 Sep; 163(9):1561-8.

5. Magnusson LL, Theorell T, Bech P, Rugulies R, Burr H, Hyde M, et al. Psychosocial working conditions and depressive symptoms among Swedish employees. Int Arch Occup Environ Health 2009 Ago; 82(8):951-60.

6. Wang JL, Patten SB. Perceived work stress and major depression in the Canadian employed population, 20-49 years old. J Occup Health Psychol 2001; 6:283-289.

7. Wang JL. Perceived work stress, imbalance between work and family/personal lives, and mental disorders. Soc Psychiatry Psychiatr Epidemiol 2006; 41:541-548.

8. Wieclaw J, Agerbo E, Mortensen PB, Burr H, Tuchsen F, Bonde JP. Psychosocial working conditions and the risk of depression and anxiety disorders in the Danish workforce. BMC Public Health 2008 Ago; $7(8): 280$.

9. Wang JL, Schmitz N, Smailes E, Sareen J, Patten S. Workplace characteristics, depression, and healthrelated. Presentteism in a General Population Sample. Am Environ Health 2010 Ago; 52(8):836-842.

10. Boles M, Pelletier B, Lynch W. The relationship between health risks and work productivity. J Occup Environ Med $2004 ; 46: 737-745$.

11. Goetzel, RZ, Long SR, Ozminkowski RJ, Hawkins K, Wang S, Lynch W. Health, absence, disability, and presenteeism cost estimates of certain physical and mental health conditions affecting U.S. employers. J Occup Environ Med 2004; 46:398-412.

12. Nieuwenhuijsen K, Verbeek JH, De Boer AG, Blonk RW, Van Dijk FJ. Predicting the duration of sickness absence for patients with common mental disorders in occupational health care. Scand J Work Environ Health 2006 Feb; 32(1):67-74.

13. Rugulies R, Bültmann U, Aust B, Burr H. Psychosocial work environment and incidence of severe depressive symptoms: prospective findings from a 5-year follow-up of the Danish work environment cohort study. Am J Epidemiol 2006 May; 163(10):877-87.

14. Demir F, Ay P, Erbaş M, Ozdil M, Yaşar E. The prevalence of depression and its associated factors among resident doctors working in a training hospital in Istanbul. Turk Psikiyatri Derg 2007; 18(1):31-7.

15. Kessler RC, Merikangas KR, Wang PS. The prevalence and correlates of workplace depression in the national comorbidity survey replication. J Occup Environ Med 2008 Abr; 50(4):381-90.

16. Wege N, Dragano N, Erbel R, Jockel KH, Moebus S, Stang A, et al. When does work stress hurt? Testing the interaction with socioeconomic position in the Heinz Nixdorf Recall Study. J Epidemiol Community Health 2008 Abr; 62(4):338-41.

17. Kouvonen A, Oksanen T, Vahtera J, Stafford M, Wilkinson R, Schneider J. Low workplace social capital as a predictor of depression: the Finnish Public Sector Study. Am J Epidemiol 2008 May; 167(10):1143-51. 
18. Sinokki M, Hinkka K, Ahola K, Koskinen S, Klaukka T, Kivimäki M, et al. The association between team climate at work and mental health in the Finnish Health 2000 Study. Occup Environ Med 2009 Ago; 66(8):523-8.

19. Wang J, Schmitz N, Dewa C, Stansfeld S. Changes in perceived job strain and the risk of major depression: results from a population-based longitudinal study.Am J Epidemiol 2009 May; 169(9):1085-91.

20. Clumeck N, Kempenaers C, Godin I, Dramaix M, Kornitzer M, Linkowski P, et al. Working conditions predict incidence of long-term spells of sick leave due to depression: results from the Belstress I prospective study. J Epidemiol Community Health 2009 Abr; 63(4):286-92.

21. Hämmig O, Gutzwiller F, Bauer G. Work-life conflict and associations with work- and nonwork-related factors and with physical and mental health outcomes: a nationally representative cross-sectional study in Switzerland. BMC Public Health 2009 Nov; 30(9):435.

22. Lo Sasso AT, Rost K, Beck A. Modeling the impact of enhanced depression treatment on workplace functioning and costs: a cost-benefit approach. Med Care 2006 Apr; 44(4):352-8.

23. Schwenk TL, Gorenflo DW, Leja LM. A survey on the impact of being depressed on the professional status and mental health care of physicians. J Clin Psychiatry 2008 Abr; 69(4):617-20.

24. Sicras A, Blanca M, Gutiérrez L, Salvatella J, Navarro R. Impacto de la morbilidad, uso de recursos y costes en el mantenimiento de la remisión de la depresión mayor en España: estudio longitudinal de ámbito poblacional. Gac Sanit 2010; 24(1):13-19.

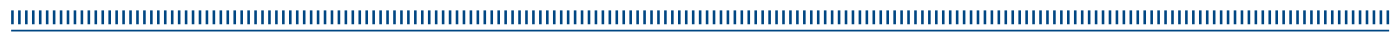

